\title{
Correspondence
}

\section{Klebsiella pneumoniae}

Sir,

We are grateful for the opportunity of replying to the letters of Drs Brewerton and Warren and Dr Eastmond, Professors Cooke, and Wright (Annals, 37, 298).

Both groups of authors point out the high incidence of Klebsiella present in hospital associated environments, on the hands of staff and in food. We were aware of these problems when our paper was submitted and would like to point out that the great majority of our patients were out patients who did not have meals in the hospital. Faecal samples were collected at home either the night before or on the morning of the visit and were handed over in a sealed specimen pot at the time of the assessment. In contrast, our control group did consist of a group of hospital associated volunteers and therefore, if anything, we have selected a control group whose findings are against us rather than in our favour. We hope to carry out a subsequent study on the faecal carriage rate in a non hospital population. We have also been studying a group of out patients with rheumatoid arthritis over the past year as a second control group and the overall faecal carriage rate of Klebsiella in this group is $45 / 205$ $(22 \%)$ a value lower than in the patients with active or probably active disease and comparable to the patients with inactive disease.

We do not have any data on the sero types of the organisms isolated and there is no clear evidence of a seasonal variation in isolation. Results of faecal cultures on patients or controls who received antibiotics were not included in our studies.

Drs Brewerton and Warren point out that the distinction between active and probably active patients is difficult and that a possible source of bias is that certain patient results may be included more than once in each particular study. We can only agree with their comments and point out that we have tried to adjust for some of these limitations by our second method of analysis where we have included both active and probably active patients into one group and where only one assessment and one faecal culture was compared at each 3 monthly interval. If the patient was assessed as active or probably active at any time during this period then an active score was given. If at any time during that 3 months a patient had a positive culture then the result was recorded as positive. Similarly, if several cultures during this period were positive it still only counted as one result. Our findings show that the combined active group had a significantly higher incidence of Klebsiella, $63 \%$ than the inactive group, $16 \%\left(\chi^{2}=78 \cdot 2\right)$.

We described in our report that we had included a small number of patients who had associated diseases such as psoriasis or inflammatory bowel disease. These patients all had sacroiliitis or spondylitis. Eighteen patients had symptoms of back pain and stiffness, peripheral joint involvement, and normal or suspicious sacroiliac joints on $x$-ray. All were B27 positive. We included these patients because we had particularly sought for young patients at the onset of their symptoms.

It may take several years for characteristic $x$-ray changes to develop at the sacroiliac joints. Several of the patients have been studied by sacroiliac scintiscanning and they show increased activity over the affected joints. We considered that on balance the inclusion of these patients was justified. Only a small number of patients had an episode of diarrhoea with an exacerbation of their disease.

In our section on sequential studies, we wish to point out that we selected all available inactive disease episodes at which a faecal culture was collected and at which an assessment of subsequent disease activity over the next three months was available. We had 196 episodes available for study in the 95 patients. On 35 occasions the cultures were positive and in 161 they were negative. An assessment of subsequent active or probably active disease was made on 41 occasions, 15 of these were in the Klebsiella positive episodes. These results are shown by our third method of comparison. Our figures show that about $15 / 35(43 \%)$ of patients who have inactive disease assessed and who have positive cultures for Klebsiella go on to develop a clinical worsening as compared to $(16 \%)$ who do not.

We do not wish to make any specific comments on the findings of Dr Eastmond and his group. It is clear that the criteria and methods used for the selection of patients into the various disease activity groups will decide whether a difference in carriage rate of Klebsiella between active and inactive disease can be confirmed.

Although we would agree in suggesting caution before attributing infection by Klebsiella as an aetiological factor in ankylosing spondylitis we believe that the crossreactivity hypothesis is the best and most comprehensive theory available at present to explain the cause of this disease and that further studies along these lines are justified.

ROLAND EBRINGER,

D. R. CAWDELL

P. Cowling and A. EBringer

The Middlesex Hospital, Arthur Stanley House, Tottenham Street, London W1P 9PG]

\section{Subtle spine abnormalities in patients with back pain: fracture $v s$. congenital anomaly}

Sir,

Sims-Williams et al. (Annals, 37, 262-265) recently reported the utilisation of stereoscopic radiography in 7 